\title{
MEASURES THAT VANISH ON HALF SPACES ${ }^{1}$
}

BENJAMIN WEISS

I. Introduction. It is well known that if $f \in L^{1}\left(E_{n}\right)$ ( $E_{n}$ denotes real Euclidean $n$-space, and all functions are complex valued) has the property that

$$
\int_{H} f(x) d x=0
$$

for all half spaces $H$, then $f(x)=0$ a.e. It is natural to conjecture that if (1) holds for all $H \in \mathcal{H}_{n}$, where $\mathfrak{H}_{n}$ is the set of all half spaces of $E_{n}$ that exclude the unit sphere, then $f(x)=0$ a.e. in $\{|x|=1\}$. Recently S. Helgason has proven this assuming the a priori estimate $f(x)$ $=O\left(|x|^{-m}\right)$ for all $m>0$ [5]. The simple example (due to D. J. Newman) of $f(x)=1 /\left(x_{1}+i x_{2}\right)^{3}$ if $|x| \geqq 1$ and zero otherwise, which by Cauchy's theorem satisfies (1) for all $H \in \mathfrak{F}_{2}$, shows that without some assumption the conjecture is in fact false.

The purpose of this note is to characterize explicitly those $f \in L^{1}\left(E_{n}\right)$ that satisfy (1) for all $H \in \mathcal{H}_{n}$. The second section is devoted to a Paley-Wiener theorem for Hankel transforms which is needed in the proof of the main result. This is found in the final section together with a few concluding remarks. Another version of a Paley-Wiener theorem for Hankel transforms may be found in [4]. I am indebted to the referee for this reference.

II. A P-W Theorem for Hankel transforms. The following theorem of Plancherel and Polya [7] will be used.

(P) If $f \in L^{1}\left(E_{n}\right)$ and $F(y)=\int_{E_{n}} f(x)$ exp $(-2 \pi i(x, y)) d x$ (or $f \in L^{2}\left(E_{n}\right)$ and $F$ its Fourier transform $)$ then $f$ vanishes a.e. in $\{|x| \geqq 1\}$ if and only if $F$ is an entire function of exponential type $2 \pi$ in every direction.

The Hankel transform of order $\nu$ is defined by

$$
F(y)=\int_{0}^{\infty} f(x) J_{\nu}(2 \pi x y)(x y)^{1 / 2} d x
$$

where $J_{\nu}$ is the Bessel function of order $\nu$ and either $f \in L^{1}(0, \infty)$ or $f \in L^{2}(0, \infty)$ and the integral is taken as l.i.m. [2, \$42].

Received by the editors, December 8, 1964.

${ }^{1}$ Presently at the IBM T. J. Watson Research Center, Yorktown Heights, New York. 
LemMA 1. If $F$ is given by (2) and $\nu=n-\frac{1}{2}(n=1,2, \cdots)$ (resp. $\nu=n)$ then $F\left(\right.$ resp. $\left.y^{1 / 2} F(y)\right)$ is an entire function of exponential type $2 \pi$ if and only if $f\left(\operatorname{resp} . x^{-1 / 2} f(x)\right)=f_{0}+\sum_{j=1}^{n} c_{j} r_{j}$ where $f_{0}(x)=0$ for $x \geqq 1, r_{j}(x)=x^{-j}$ if $x \geqq 1$ and zero otherwise, and the $c_{j}$ 's are constants.

Proof. The proof for $\nu=n$ is just like the proof for $\nu=n-1 / 2$ and so we confine our attention to this latter case. Furthermore, it is quite straightforward to reduce the case of $f \in L^{1}$ to $f \in L^{2}$ and thus it suffices to prove the lemma assuming that $f \in L^{2}$.

(a) Suppose first that $F$ has a zero of order $\geqq n$ at $y>0$. Then $G(y)=F(y) / y^{n}$ is also an entire function of exponential type $2 \pi$ and setting $g(x)=f(x) / x^{n}$ we have

$$
G(y)=y^{-n+1 / 2} \int_{0}^{\infty} g(x) J_{\nu}(2 \pi x y) x^{n+1 / 2} d x .
$$

If $g$ is considered as a radial function in $L^{2}\left(E_{2 n+1}\right)$ then except for a factor of $2 \pi$ the right hand side of (3) gives the Fourier transform of $g$ $[1, \S 2.6]$. Applying (P) we conclude that in this case $f$ itself vanishes for $x \geqq 1$.

(b) If $r_{j}$ is inserted for $f$ in (2) the corresponding $R_{j}$ are given by

$$
R_{j}=y^{j-1} \int_{0}^{\infty} J_{\nu}(2 \pi x) x^{-j+1 / 2} d x+S_{j}
$$

where $S_{j}$ is an entire function of exponential type $2 \pi$ and vanishes at zero to order $\geqq n$. From (a) we see that the first term cannot vanish and hence if a suitable linear combination of $r_{j}(j=1,2, \cdots, n)$ is subtracted from $f$ we are reduced to the situation in (a), and this completes the proof in one direction. The other direction is immediate since $J_{\nu}$ is entire of exponential type $2 \pi$, a fact which we used in our assertion about $S_{j}$.

The following is an immediate consequence of the lemma and $(P)$.

Corollary. If $f \in L^{1}\left(E_{n}\right)$ is a radial function $(f(x)=\tilde{f}(|x|))$ and

$$
\tilde{F}(y)=2 \pi i^{k} y^{-n} \int_{0}^{\infty} \tilde{f}(w / y) J_{k+(n-1) / 2}(2 \pi w) w^{n / 2} d w,
$$

then $F$ is entire of exponential type $2 \pi$ if and only if $f=f_{0}+\sum_{j=1}^{x-1} c_{j} r_{j}$ $(n \geqq 2, k>0)$.

\section{The main theorem. Let}

$$
A_{n}=\left\{f \epsilon L^{1}\left(E_{n}\right):(1) \text { holds for all } H \in \mathfrak{H}_{n}\right\} .
$$

Observe that (i) $A_{n}$ is a closed subspace of $L^{1}\left(E_{n}\right)$, and (ii) $A_{n}$ is rota- 
tion invariant in the sense that $R \in S O(n)$ and $f \in A_{n}$ implies that $R f \in A_{n}(R f(x)=f(R x))$. Denote by $\sum f_{k}\left(|x|, x^{\prime}\right)\left(x^{\prime}=x /|x|\right)$ the expansion of $f$ in spherical harmonics, i.e., for fixed $|x|, f_{k}$ is a spherical harmonic of degree $k[1, \S 2.7]$. The main reduction is accomplished by

Lemma 2. If $f \in L^{1}\left(E_{n}\right)$ then $f \in A_{n}$ if and only if $f_{k} \in A_{n}$ for all $k$.

Proof. Assume first that $f \in A_{n}$, then $f_{k}$ may be expressed as

$$
f_{k}\left(|x|, x^{\prime}\right)=\int_{\text {so }(n)} R f(x) Z_{k}\left(R y^{\prime}, y^{\prime}\right) d R
$$

where $y^{\prime}$ is a fixed unit vector and $Z_{k}$ is a zonal harmonic of degree $k$ $[3, \mathrm{XI}]$. Since translation is continuous in the $L^{1}$ norm, one verifies easily that $R \rightarrow R f$ is a continuous map from $S O(n) \rightarrow L^{1}\left(E_{n}\right)$ and it then follows from (i) and (ii) that $f_{k} \in A_{n}$.

Conversely, if $f_{k} \in A_{n}$ for all $k$ then so are the appropriate Abel means of $\sum f_{k}$. Now the Abel means of a continuous function converge [6] $]^{2}$ and thus by (i) $f \in A_{n}$.

Next we identify the Fourier transform of $A_{n}$ in

Lemma 3. If $f \in L^{1}\left(E_{n}\right)$ and $F(y)=\int_{E_{n}} f(x) \exp (-2 \pi i(y, x)) d x$ then $f \in A_{n}$ if and only if for all $t \in E_{n}$ with $|t|=1$ we have that $F_{t}$ is an entire function of exponential type $2 \pi$ where $F_{t}(z)=F\left(t_{1} z, t_{2} z, \cdots, t_{n} z\right)$.

Proof. By (ii) it suffices to consider $t=(1,0, \cdots, 0)$. Fubini's theorem yields

$$
\begin{aligned}
F_{t}(z) & =\int_{E_{n}} f(x) \exp \left(-2 \pi i z x_{1}\right) d x \\
& =\int_{E_{1}} \exp \left(-2 \pi i z x_{1}\right)\left\{\int_{E_{n-1}} f(x) d x_{2} \cdots d x_{n}\right\} d x_{1} .
\end{aligned}
$$

Since $F \in A_{n},\{\}$ as a function of $x_{1}$ vanishes for $\left|x_{1}\right| \geqq 1$ and thus $F_{t}$ is entire of exponential type $2 \pi$. The converse follows from (5) and (P).

TheOREM. If $f \in L^{1}\left(E_{n}\right)$ and $f_{k}$ are given by (4) then $f \in A_{n}$ if and only if

$$
f_{k}\left(|x|, x^{\prime}\right)=f_{k, 0}\left(|x|, x^{\prime}\right)+\sum_{j=1}^{k-1} c_{k, j}\left(x^{\prime}\right) r_{k+j}(|x|)
$$

2 The theorem is given there for real valued continuous functions but may be easily extended to continuous functions with values in a Banach space (here $L^{1}$ ). 
where $f_{k, 0}$ vanishes for $|x| \geqq 1 . c_{k, j}$ are harmonics of degree $k$ (as is $f_{k, 0}$ for fixed $|x|)$ and $r_{m}$ is defined in Lemma 1.

Proof. If $F_{k}$ denotes the Fourier transform of $f_{k}$ then

$$
F_{k}\left(|y|, y^{\prime}\right)=2 \pi i^{k}|y|^{-n} \int_{0}^{\infty} f_{k}\left(w /|y|, y^{\prime}\right) J_{k+(n-1) / 2}(2 \pi w) w^{n / 2} d w
$$

$[2, \S 2.7]$. The theorem now follows from Lemma $2-3$ and the corollary of Lemma 1.

Helgason's result is obtained upon noticing that his a priori bound on $f$ carries over to $f_{k}$ and implies that the $c_{k, j}$ are identically zero. The results of this note carry over mutatis mutandis to measures, the details are omitted.

Acknowledgment. I thank Professor E. M. Stein for his advice and helpful suggestions in the preparation of this note.

\section{BIBLIOGRAPHY}

1. S. Bochner, Harmonic analysis and the theory of probability, Univ. of California Press, Berkeley and Los Angeles, Calif., 1960.

2. - Lectures on Fourier integrals, Annals of Mathematics Studies No. 42, Princeton Univ. Press, Princeton, N. J., 1959.

3. A. Erdelyi, et al., Higher transcendental functions, Vol. 2, McGraw-Hill, New York, 1953.

4. J. L. Griffith, Hankel transforms of functions zero outside a finite interval, J. Proc. Roy. Soc. New South Wales 89 (1956), 109-115.

5. S. Helgason, The Randon transform on Euclidean spaces, compact two-point homogenous spaces and Grassman manifolds, Acta Math. 113 (1965), 153-180.

6. T. Kubota, Ueber die Konvex-geschlossenen Mannigfaltigkeiten in n-dimensionale Raume, Sci. Rep. Tôhoku Imperial Univ. 14 (1925), 85-99.

7. M. Plancherel and G. Polya, Fonctions entières et integral de Fourier multiples, Comment Math. Helv. 9 (1936-1937), 224-248.

Princeton University 